\title{
DIFICULTADES DE LOS ESTUDIANTES EN LA ADAPTACIÓN DE LA METODOLOGÍA DE EDUCACIÓN A DISTANCIA EN LA UNIVERSIDAD NACIONAL ABIERTA Y A DISTANCIA UNAD, CERES VILLA DE SAN SEBASTIÁN DE LA PLATA, HUILA
}

\author{
Alejandro Atehortúa Marulanda ${ }^{6} y$ \\ Ángel Adriana Liscano ${ }^{7}$
}

\begin{abstract}
RESUMEN
La Universidad Nacional Abierta y a Distancia UnAD, Ceres Villa de San Sebastián de La Plata, departamento del Huila oferta un modelo de educación a distancia que se descentraliza para llegar a todo el país, convirtiéndose en una oportunidad de fácil acceso a la educación superior, que busca contribuir al mejoramiento de la calidad de vida de todas y cada una de las regiones a las cuales llega.
\end{abstract}

Sin embargo, por ser un modelo innovador en el sector educativo, se presentan dificultades de insatisfacción en los estudiantes que acuden a este centro educativo. Esta situación motivó el desarrollo de una investigación con el objetivo de identificary describir las principales dificultades de los estudiantes en la adaptación al sistema de educación a distancia de la Universidad Nacional Abierta y a Distancia UNAD. Se aplicó el método de enfoque cuantitativo de tipo descriptivo, haciendo uso de técnicas e instrumentos como la observación, la entrevista y la encuesta a líderes de las escuelas. El estudio encontró dificultades significativas en los estudiantes tales como la procedencia de un sistema presencial, la insatisfacción con la formación que reciben de la Universidad, la dificultad con los costos de matrícula y otros derivados del desarrollo del proceso educativo. La investigación mostró que el tiempo destinado a la inducción es insuficiente, que los programas básicos de informática no operan completamente, tampoco cuentan con fácil acceso a Internet en su lugar de residencia. Los retrasos en las tutorías por cruce de horarios y la no contratación a tiempo de los asesores, entre otras, son también aspectos que afectan el proceso educativo; situación reconocida por los administrativos y directivos de la UNAD. De otro lado, la falta de prácticas que permitan contextualizar la teoría es otra dificultad relevante no sólo para los estudiantes sino también para las directivas del Ceres, Villa de San Sebastián de La Plata. En este estudio, el programa de Psicología de la Escuela de Ciencias Sociales, Artes y Humanidades presentó más dificultades.

La investigación evidencia que existen serias dificultades en la adaptación al sistema de educación a distancia, lo que podría ser la causa de los fracasos académicos reflejados en la pérdida de cursos, bajas calificaciones, cambio de universidad, y deserción universitaria.

\footnotetext{
6 CERES La Plata, Líder Grupo de Investigación Koküa, tutor ECSAH. E-mail: alejoth4@yahoo.com

7 Nombre del semillero: Innovación READSI, Línea de investigación: Educación a Distancia. E-mail: angela.alizcano@yahoo.es
} 
Palabras clave: metodología, sistema, distancia, flexible, autoaprendizaje autónomo, presencial, adaptación, asimilación, tradicional, virtual, educación, tecnología, información, comunicación, redes, interfaces, medios, mediaciones.

\begin{abstract}
The open and distant university UNAD, Ceres Villa de San Sebastian de La Plata, in Huila, offers a distance educational model whose presence is decentralized to reach across the country, becoming an opportunity for easy access to higher education that seeks to contribute to the improvement of quality of life of population in every region of the country.
\end{abstract}

Since it is an innovative model in the education sector, there are a lot of difficulties of dissatisfaction in students attending this institution. This situation let to the development of a research with the aim of identifying and describing the main students' difficulties when trying to adapt to the distance education system at the ODL UNAD. The research method applied in this study was the descriptive - quantitative approach, in which it was used techniques and instruments such as observation, interview and survey to the school leaders. The methodological procedures let find significant difficulties in students coming from the presence educational system. Most of them report dissatisfaction with the university training, economical difficulties for paying tuition and costs related to the development of the educational process.

The research showed, among others, that the time for the induction to this distance system was not enough, also that students do not use, or operate completely basic computer programs, and that they do not have easy access to Internet at home. The tutoring strategy is not efficient due to problems with schedule; situation that was accepted by the staff of the university. On the other hand, the lack of practice that allow to contextualize the theory with the practice was another relevant finding, not only for the students but also for the managers of the Ceres, Villa de San Sebastian La Plata

According to this study there are serious difficulties in the students' adaptation to the distance education system, which should call the attention for seeking solution because it could be the cause of academic failure reflected sometimes on the loss of courses, poor results, or university desertion, among others.

Key words: Methodology, systems, distance, flexible, self-learning, presential (face to face) instruction, adaptation, assimilation, traditional, virtual, education, technology, information, communication, networks, interfaces, media, mediation.

\title{
INTRODUCCIÓN
}

El presente trabajo denominado Dificultades de los estudiantes en la adaptación al sistema de la Educación a Distancia de la Universidad Nacional Abierta y a Distancia UNAD CERES Villa de San Sebastián de La Plata, identifica las principales dificultades que tienen los 
estudiantes en el proceso de su formación tecnológica y profesional en el Centro en donde se tomó la población objeto de estudio consistente en 313 estudiantes. Se seleccionó como muestra aleatoria estratificada un total de 118 estudiantes de las diferentes Escuelas que oferta la Universidad en la región.

Se utilizaron como técnicas la observación a tutorías, la encuesta a los estudiantes de cada uno de los Programas, y entrevistas a directivos, administrativos, a la consejera académica del Centro y a los tutores.

Los resultados que arroja la investigación tienen que ver con el tiempo insuficiente destinado a la inducción, el no uso permanente y apropiado de las estrategias metodológicas propias de la Universidad por parte de estudiantes y tutores, las dificultades económicas y personales de los educandos y la deficiencia en la conectividad, entre otros. El trabajo evidencia dificultades de gran importancia dentro de la prestación del servicio a los estudiantes y constituye un aporte al diagnóstico de las necesidades e intereses de la comunidad Unadista, en su proceso de formación tecnológica y profesional en la Universidad Nacional Abierta y a Distancia UNAD.

Actualmente, la UNAD Ceres Villa de San Sebastián de La Plata, Huila, tiene 313 estudiantes matriculados en los diferentes programas que ofrece la sede en este municipio, a través de cinco escuelas: Escuela de Ciencias Agrícolas, Pecuarias y del Medio Ambiente, Escuela de Ciencias Sociales, Artes y Humanidades Escuela de Ciencias Básicas, Tecnología e Ingeniería, Escuela de Ciencias Administrativas, Contables, Económicas y de Negocios y Escuela de Ciencias de La Educación.

Por el sistema tradicional, los estudiantes en general, acuden cada 15 días a las tutorías; desarrollan y presentan avances de la guía de actividades académicas programadas para el curso, orientadas por los docentes denominados tutores. A su vez, por el campus virtual, los estudiantes tienen acceso a módulos con contenidos didácticos. Los procesos de aprendizaje y el acompañamiento tutorial se realiza por la plataforma virtual.

Sin embargo, se presentan situaciones de insatisfacción por parte del estudiante que dificultan su proceso de formación académica y profesional: la inasistencia a las tutorías, resultados deficientes en su proceso de aprendizaje, reflejados en las bajas calificaciones de evaluaciones parciales y nacionales, que en ocasiones conlleva a la pérdida de los cursos académicos, hecho que se visualiza en cartelera del Centro, y en la deserción académica. De igual manera, la inconformidad de los estudiantes frente a la gestión de la Universidad en el mejoramiento de sus condiciones académicas.

La comunicación entre estudiantes y tutores es insuficiente e inadecuada y la respuesta o soluciones pertinentes a las diferentes inquietudes y necesidades del estudiante, por parte de la Universidad, no es, en la mayoría de los casos, satisfactoria; la planificación en la programación de los cursos académicos y su ajuste al plan de estudios de cada programa es improcedente. 
Otro problema identificado es la discontinuidad que presentan los estudiantes en sus procesos y la procedencia del sistema presencial practicado desde la básica primaria.

Lo anterior llevó al grupo de investigación a formularse la pregunta de investigación:

¿Cuáles son las principales dificultades de los estudiantes en la adaptación al sistema de educación a distancia de la Universidad Nacional Abierta y a Distancia UNAD Ceres Villa de San Sebastián de La Plata, Huila?

\section{OBJETIVO GENERAL}

Identificar y describir las principales dificultades que presentan los estudiantes en la adaptación al sistema de educación a distancia en la Universidad Nacional Abierta y a Distancia UNAD Ceres Villa de San Sebastián de La Plata, Huila.

\section{OBJETIVO ESPECÍFICOS}

Determinar el número de estudiantes matriculados por programa en la Universidad Nacional Abierta y a Distancia UNAD Ceres La Plata y su sitio de procedencia.

Identificar el programa en el que los estudiantes presentan mayor dificultad de adaptación en la UNAD Ceres La Plata, Huila.

Determinar las dificultades de adaptación y permanencia de los estudiantes en la Universidad Nacional Abierta y a Distancia UNAD Ceres La Plata, Huila.

Evaluar el sistema de inducción que realizó la UNAD Ceres La Plata, Huila a los estudiantes y tutores.

Evaluar el desempeño del tutor frente a la orientación del curso académico en el sistema de Educación a Distancia en la Universidad Nacional Abierta y a Distancia UNAD, Ceres La Plata, Huila.

Identificar los tipos de medios y mediaciones utilizados en la implementación del modelo de educación a Distancia en La Universidad Nacional Abierta y a Distancia UNAD, Ceres La Plata, Huila.

\section{METODOLOGÍA}

\section{Tipo de estudio}

La investigación se ubicó en el enfoque cuantitativo. Es de tipo descriptivo, por cuanto tiene como objetivo principal identificar y describir un problema específico, en este caso, Las dificultades que tienen los estudiantes en la adaptación al sistema de educación a distancia de la UNAD del municipio de La Plata, Huila. 


\section{Población:}

La población objeto de estudio está integrada por 313 estudiantes matriculados, provenientes de diferentes municipios del Huila y del oriente del Cauca, distribuidos en los diferentes programas y especializaciones, ofrecidos por la Universidad en cada una de las escuelas así:

- Escuela de Ciencias Agrícolas, Pecuarias y del Medio Ambiente 103

- Escuela de Ciencias Sociales, Artes y Humanidades 55

- Escuela de Ciencias Básicas, Tecnología e Ingeniería 100

- Escuela de Ciencias Administrativas, contables

- Económicas y de Negocios 46

- Escuela de Ciencias de La Educación

\section{Muestra:}

La muestra se seleccionó de forma aleatoria estratificada, la cual se agrupó por programas y en cada programa se seleccionaron de manera aleatoria los estudiantes.

\section{Métodos:}

Para el desarrollo de la investigación se utilizaron los métodos, técnicas e instrumentos que a continuación se describen.

\section{Fase 1. Observación exploratoria de la realidad}

Observación Natural: a través de ella se buscaba hacer un acercamiento con el objeto de estudio (comunidad educativa) con la intención de hacer una exploración de la realidad e identificar un problema o situación que ameritara la investigación.

Para tal efecto se aplicó, observación participativa con la comunidad académica, con el objetivo de ampliar la información anteriormente recolectada.

\section{Fase 2. Aplicación de la prueba piloto}

Diseño de la encuesta: se diseñó con el ánimo de identificar y evidenciar las dificultades, que los estudiantes habían manifestado. La encuesta fue semiestructurada a fin de que permitiera obtener datos de acuerdo con el tipo de variables. Una vez se diseñó el instrumento, se procedió a la aplicación de la prueba piloto a los estudiantes de los programas ofertados por la UNAD, a partir de la cual se hicieron las correcciones con el objeto de garantizar la validez del instrumento.

Aplicación: en la aplicación de la primera encuesta, participaron dos de las integrantes del proyecto, ubicando a los estudiantes en el Ceres Villa de San Sebastián de La Plata, a quienes se les dio la encuesta, respondieron y entregaron a las investigadoras.

Análisis: se evidenció de manera general que los estudiantes sí presentaban dificultades en la metodología a distancia, sin embargo, se observó que la encuesta por contener preguntas 
abiertas, no logró identificar las dificultades de manera precisa. Por tal motivo, se determinó realizar una segunda encuesta estructurada, que nos permitiera identificar con exactitud las dificultades.

Diseño de la entrevista: se utilizó un guión con punteo con el fin de concertar la información incompleta en la encuesta. La aplicación fue realizada a estudiantes, tutores y a la funcionaria de registro y control.

\section{Fase 3. Diseño y aplicación}

Observación: se realizó con el fin de corroborar la orientación del curso académico en el sistema de educación a distancia, estructurada en nueve (9) ítems y aplicada a las tutorías presenciales, para evidenciar el manejo de la tutoría por parte del tutor, la metodología y la empatía, entre otras.

Encuesta: a partir de la prueba piloto se estableció la realización de otra encuesta estructurada, en cinco (5) ítems, treinta y tres (33) preguntas, que permitieron ampliar la información recolectada; sin embargo, esta encuesta no dio respuesta a algunos de los objetivos específicos planteados en el proyecto. Por lo que se hizo necesario una nueva encuesta estructurada de veintitres (23) preguntas que tenían como objetivo evidenciar las dificultades de los estudiantes en la adaptación de la metodología a distancia.

Entrevista: a partir de la información recolectada se planteó la realización de una entrevista dirigida a la comunidad académica: directora, administrativos, consejera y estudiantes con el fin de confrontar la información obtenida a través de las anteriores técnicas descritas.

\section{Tabulación, Análisis E Interpretación De Los Resultados Consolidado}

\section{Ítem 1. Información personal}

Figura 1. Género de los estudiantes encuestados

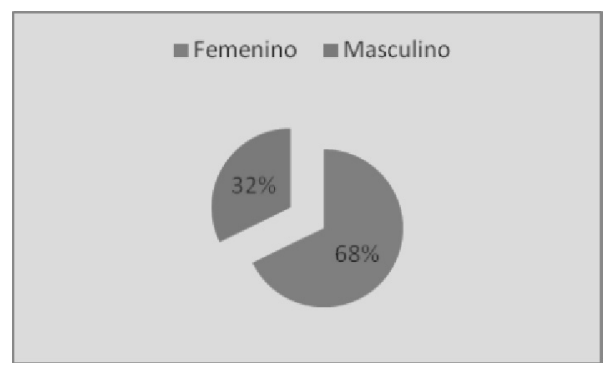

\begin{tabular}{|lcc|}
\hline Variable & hi & HI \\
\hline Femenino & 80 & $68 \%$ \\
\hline Masculino & 38 & $32 \%$ \\
\hline TOTAL & 118 & $100 \%$ \\
\hline
\end{tabular}

De 118 estudiantes encuestados en la UNAD Ceres La Plata, el 68\% pertenece al género femenino y el $32 \%$ corresponde al género masculino. 
Figura 2. Lugar de residencia de los encuestados

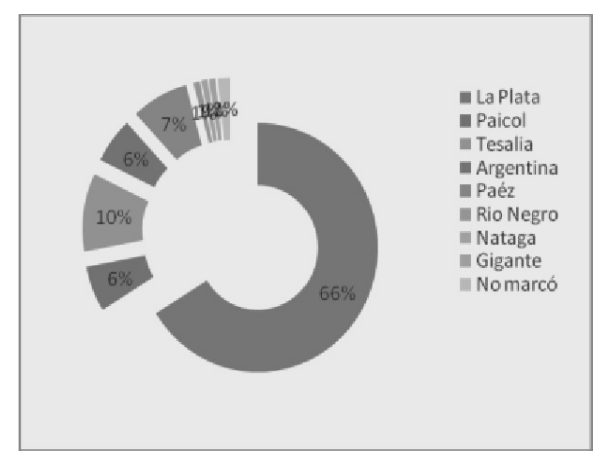

\begin{tabular}{|lcc|}
\hline Variable & hi & HI \\
\hline La Plata & 78 & $66 \%$ \\
\hline Paicol & 7 & $6 \%$ \\
\hline Tesalia & 12 & $10 \%$ \\
\hline Argentina & 7 & $6 \%$ \\
\hline Páez & 9 & $8 \%$ \\
\hline Rio Negro & 1 & $1 \%$ \\
\hline Nátaga & 1 & $1 \%$ \\
\hline Gigante & 1 & $1 \%$ \\
\hline No marcó & 2 & $2 \%$ \\
\hline Total & 118 & $100 \%$ \\
\hline
\end{tabular}

Del 100\% de los estudiantes de la UnAD Ceres La Plata, el 66\% reside en el municipio de La Plata, Huila, el 10\% en Tesalia, el 8\% en Páez, el 6\% en Paicol, otro 6\% en La Argentina, el $2 \%$ no marcó ninguna opción y con el $1 \%$ cada uno en los municipios de Nátaga, Gigante y Río Negro.

Lo anterior evidencia la forma como está integrada la familia Unadista, es decir, por estudiantes, en su gran mayoría del lugar donde se ubica el Ceres, y también de otros municipios aledaños a la sede principal.

\section{Ítem 2. Información académica}

Figura 3. Es egresado de una institución con modalidad

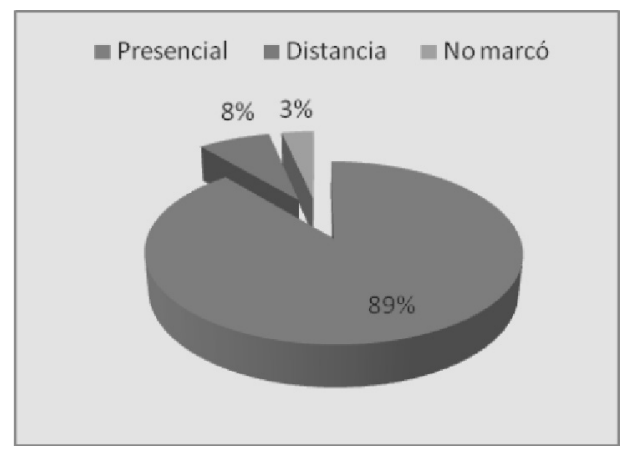

\begin{tabular}{|lcc|}
\hline Variable & hi & HI \\
\hline Presencial & 105 & $89 \%$ \\
\hline Distancia & 9 & $8 \%$ \\
\hline No marcó & 4 & $3 \%$ \\
\hline TOTAL & 118 & $100 \%$ \\
\hline
\end{tabular}

El $89 \%$ de los estudiantes de la Universidad Nacional Abierta y a Distancia, UNAD, son egresados de una institución con modalidad presencial, el $8 \%$ proviene de una institución con modalidad a distancia y el 3\% no marcó ninguna opción. Con ello se muestra que 
la procedencia de los estudiantes de la UNAD, es de un sistema presencial, que posee una marcada diferencia en el aprendizaje a distancia.

Figura 4. La mayoría de los cursos académicos los realiza por el sistema

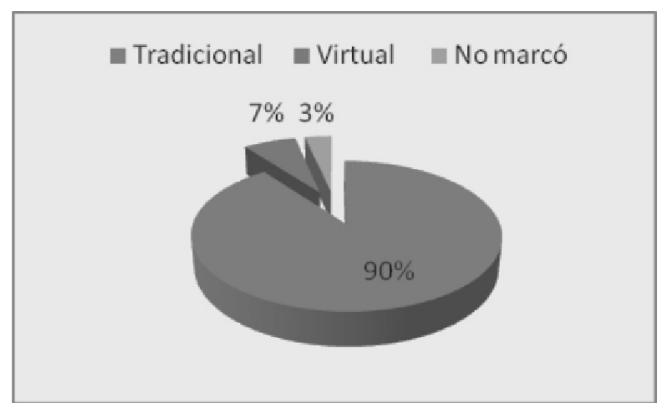

\begin{tabular}{|lll|}
\hline \multicolumn{1}{|c|}{ Variable } & \multicolumn{1}{c|}{ hi } & \multicolumn{1}{c|}{ HI } \\
\hline Tradicional & 106 & $90 \%$ \\
\hline Virtual & 8 & $7 \%$ \\
\hline No marcó & 4 & $3 \%$ \\
\hline TOTAL & 118 & $100 \%$ \\
\hline
\end{tabular}

El 90\% de los estudiantes encuestados del Ceres La Plata, Huila realiza, por el sistema tradicional, la mayoría de sus cursos académicos; el 7\% por el sistema virtual y el 3\% no marcó opción alguna. Se evidencia que los estudiantes tienen preferencia por el sistema tradicional en la educación a distancia instituido en la UNAD, es decir, que aún no se encuentra familiarizado con la metodología a distancia por la mediación virtual.

Figura 5. El sistema seleccionado fue por decisión

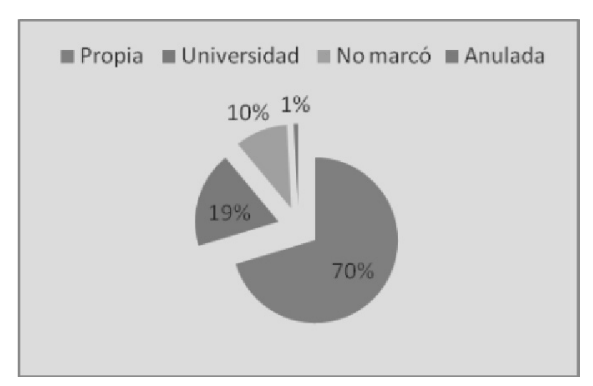

\begin{tabular}{|lcc|}
\hline \multicolumn{1}{|c}{ Variable } & hi & HI \\
\hline Propia & 83 & $70 \%$ \\
\hline Universidad & 22 & $19 \%$ \\
\hline No marcó & 12 & $10 \%$ \\
\hline Anulada & 1 & $1 \%$ \\
\hline TOTAL & 118 & $100 \%$ \\
\hline
\end{tabular}

Del 100\% de los estudiantes encuestados en la UNAD Ceres La Plata, el 70\% elige por qué mediación pedagógica matricular sus cursos académicos. El 19\% manifiesta que la Universidad decide la mediación pedagógica de sus cursos, el 10\% no marcó ninguna opción y el $1 \%$ fue anulado. Se evidencia que los estudiantes no pueden decidir por cuál sistema ver sus cursos académicos, lo que incide en una dificultad. 


\section{Ítem 3. Motivación}

Figura 6. ¿Qué motivó su ingreso a la UNAD?

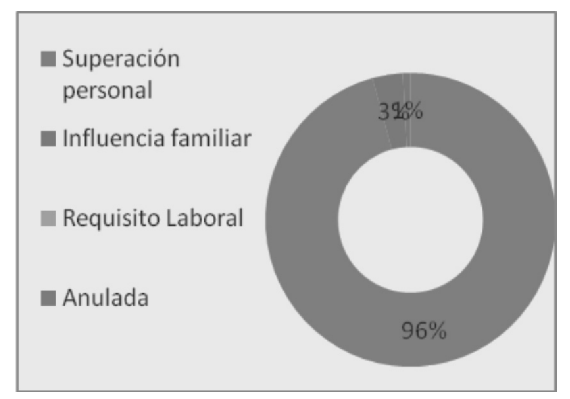

\begin{tabular}{|lcc|}
\hline \multicolumn{1}{|c}{ Variable } & hi & HI \\
\hline Superación personal & 113 & $96 \%$ \\
\hline Influencia familiar & 4 & $3 \%$ \\
\hline Requisito Laboral & - & - \\
\hline Anulada & 1 & $1 \%$ \\
\hline TOTAL & 118 & $100 \%$ \\
\hline
\end{tabular}

De los 118 estudiantes encuestados de la UNAD Ceres La Plata, el 96\% expresa que su ingreso a la UNAD responde a su deseo de superación personal, el 3\% manifiesta que se debe a la influencia familiar y el $1 \%$ fue anulado. Se evidencia que los estudiantes ingresan a la universidad con el ánimo de construir una mejor calidad de vida.

Figura 7. Está satisfecho con la formación académica que le brinda la UNAD

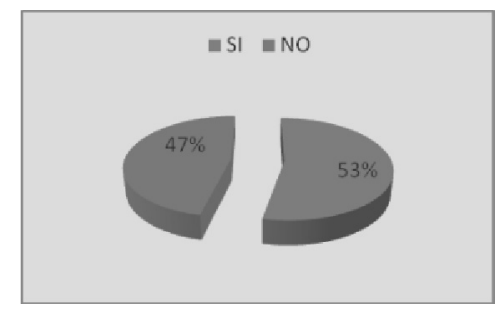

\begin{tabular}{|lcc|}
\hline Variable & hi & HI \\
\hline SI & 63 & $53 \%$ \\
\hline NO & 55 & $47 \%$ \\
\hline TOTAL & 118 & $100 \%$ \\
\hline
\end{tabular}

Del 100\% de los estudiantes encuestados de la UNAD Ceres La Plata, el 53\% está satisfecho con la formación académica que le brinda la UNAD, el 47\% está insatisfecho con la formación académica recibida. Se evidencia que un alto porcentaje de los estudiantes manifiesta no sentirse satisfecho con la formación académica, situación que dificulta la continuidad del estudiante en la Universidad. 
Figura 8. ¿Tiene dificultades en la adaptación a la metodología a distancia por influencia del sistema tradicional?

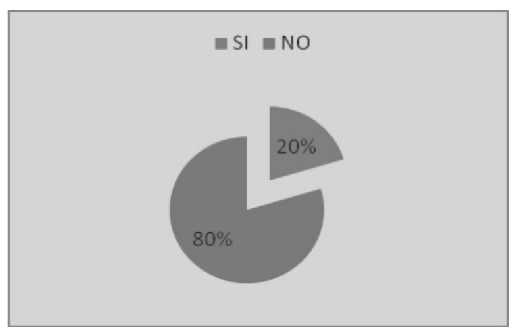

\begin{tabular}{|lcc|}
\hline Variable & hi & HI \\
\hline SÍ & 24 & $20 \%$ \\
\hline NO & 94 & $80 \%$ \\
\hline TOTAL & 118 & $100 \%$ \\
\hline
\end{tabular}

El $80 \%$ de los encuestados de la UNAD Ceres La Plata, no tiene dificultades por el sistema tradicional y el $20 \%$ manifiesta si tener dificultades por este sistema.

Figura 9. ¿Tiene dificultades en la adaptación a la metodología a distancia por sistema virtual?

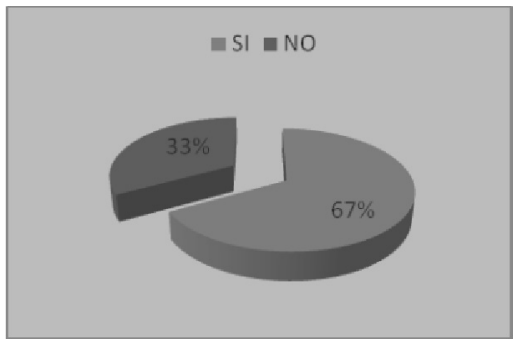

\begin{tabular}{|lcc|}
\hline Variable & hi & HI \\
\hline SÍ & 79 & $67 \%$ \\
\hline NO & 39 & $33 \%$ \\
\hline TOTAL & 118 & $100 \%$ \\
\hline
\end{tabular}

El 67\% de los estudiantes de la UNAD Ceres La Plata, tiene dificultades en la adaptación a la metodología por el sistema virtual y el 33\% manifiesta no presentar dificultad alguna de adaptación a este sistema.

Se evidencia en general que los estudiantes de la UNAD Ceres La Plata no tienen dificultades de adaptación por el sistema tradicional, sin embargo, los estudiantes que tienen cursos por el sistema virtual sí presentan dificultad.

Figura 10. ¿Considera que los actuales costos de matrícula y otros, dificultan la continuidad de sus estudios en la UNAD?

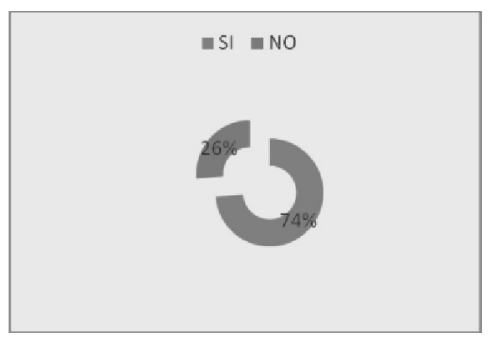

\begin{tabular}{|lcc|}
\hline Variable & hi & HI \\
\hline SÍ & 87 & $74 \%$ \\
\hline NO & 31 & $26 \%$ \\
\hline TOTAL & 118 & $100 \%$ \\
\hline
\end{tabular}


Del $100 \%$ de los estudiantes de la UNAD Ceres La Plata, el 74\% considera que los actuales costos de matrícula y otros dificultan la continuidad de sus estudios en la UNAD, mientras que el 26\% afirma no representar una dificultad para dar continuación a sus estudios.

Figura 11. ¿Tiene dificultades personales que lo lleven a suspender sus estudios?

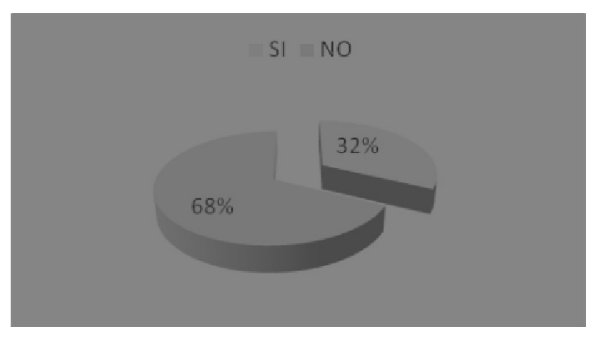

\begin{tabular}{|lcc|}
\hline Variable & hi & HI \\
\hline SÍ & 38 & $32 \%$ \\
\hline NO & 80 & $68 \%$ \\
\hline TOTAL & 118 & $100 \%$ \\
\hline
\end{tabular}

De los 118 estudiantes encuestados de la UNAD Ceres La Plata, el 68\% expresa no tener dificultades personales que lo lleven a suspender sus estudios y el 32\% tiene motivos personales que dificultan la continuidad de sus estudios de pregrado.

\section{Ítem 4. Inducción}

Figura 12. ¿Asistió al proceso de inducción que la UNAD sede La Plata efectuó al inicio de sus actividades académicas?

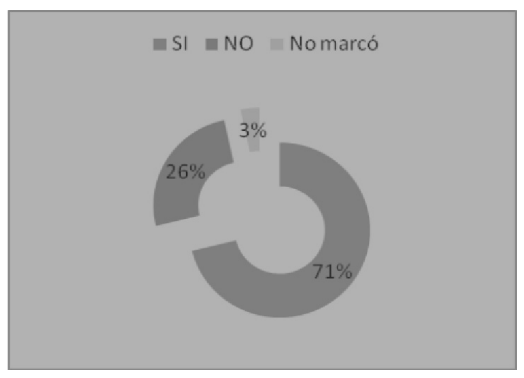

\begin{tabular}{|lcc|}
\hline Variable & hi & HI \\
\hline Sí & 84 & $71 \%$ \\
\hline NO & 30 & $25 \%$ \\
\hline No marcó & 4 & $3 \%$ \\
\hline TOTAL & 118 & $100 \%$ \\
\hline
\end{tabular}

E1 71\% de los estudiantes del Ceres asistió al proceso de inducción que la universidad efectuó al inicio de sus actividades académicas, el 25\% no asistió y el 3\% no marcó ninguna opción. Se evidencia poco interés del estudiante para apropiarse de la metodología a distancia, demostrado en inasistencia a la inducción, situación que se convierte en una dificultad. 
Figura 13. ¿Cree que el tiempo destinado para la inducción es suficiente para conocer para conocer sobre la metodología a distancia?

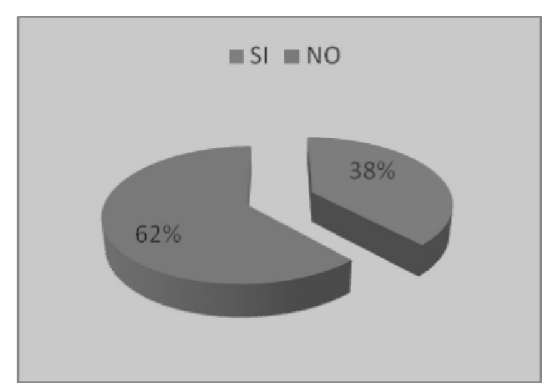

\begin{tabular}{|lcc|}
\hline Variable & hi & HI \\
\hline SÍ & 45 & $38 \%$ \\
\hline NO & 73 & $62 \%$ \\
\hline TOTAL & 118 & $100 \%$ \\
\hline
\end{tabular}

De los estudiantes encuestados del Ceres La Plata, Huila, el 62\% considera que el tiempo de inducción es insuficiente para conocer sobre la metodología a distancia y el 38\% manifiesta que sí es suficiente. Lo que indica, según los estudiantes, que el tiempo destinado para la inducción no es suficiente para conocer la metodología a distancia.

\section{Ítem 5. Aprendizaje}

Figura 14. Considera que hay mayor aprendizaje cuando trabaja

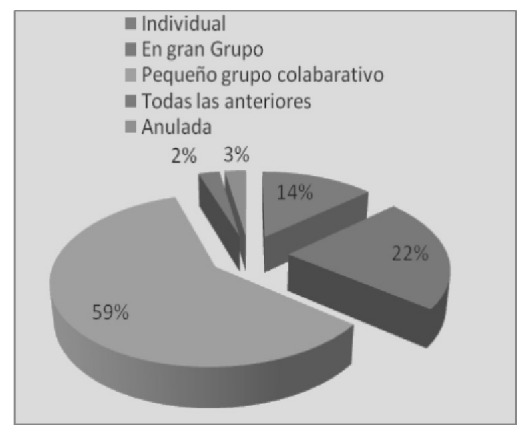

\begin{tabular}{|lll|}
\hline \multicolumn{1}{|c}{ Variable } & hi & \multicolumn{1}{c|}{ HI } \\
\hline Individual & 16 & $14 \%$ \\
\hline En gran Grupo & 26 & $22 \%$ \\
\hline Pequeño grupo colaborativo & 70 & $59 \%$ \\
\hline Todas las anteriores & 3 & $3 \%$ \\
\hline Anulada & 3 & $3 \%$ \\
\hline TOTAL & 118 & $100 \%$ \\
\hline
\end{tabular}

Del 100\% de los estudiantes encuestados de la UNAD Ceres La Plata, el 59\% manifiesta que hay mayor aprendizaje cuando trabaja en pequeño grupo colaborativo, el $22 \%$ considera que se aprende más en el gran grupo, el 14\% indica que se apropia más del conocimiento trabajando de forma individual, el 3\% expresa que todas las anteriores son importantes y otro $3 \%$ anuló su respuesta.

Es de resaltar que el estudiante tiene preferencia por el trabajo en pequeños grupos colaborativos porque considera que aprende más y muestra que en el trabajo que se desarrolla en gran grupo (tutorías), no se profundiza. 
Figura 15. ¿Utiliza y opera completamente los programas básicos de informática?

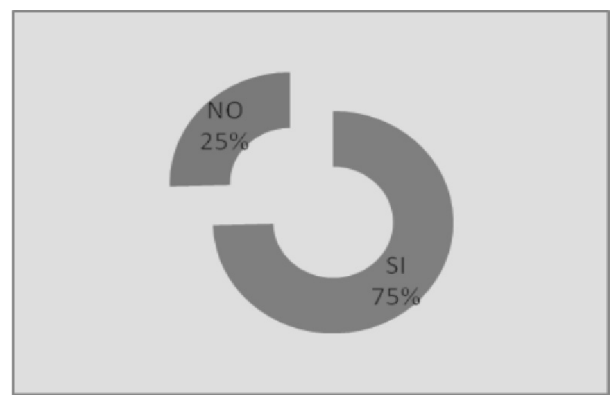

\begin{tabular}{|lcc|}
\hline Variable & hi & HI \\
\hline SÍ & 88 & $75 \%$ \\
\hline NO & 30 & $25 \%$ \\
\hline TOTAL & 118 & $100 \%$ \\
\hline
\end{tabular}

El 75\% de los estudiantes de la UNAD Ceres La Plata, utiliza y opera completamente los programas básicos de informática y el $25 \%$ no los utiliza ni opera completamente.

Se demuestra que la mayor parte de los estudiantes utiliza y opera los programas básicos de informática, sin embargo, se evidencia que hay dificultad en alto porcentaje de estudiantes que no manejan completamente los programas básicos de informática.

Figura 16. ¿ La plataforma virtual está habilitada cada vez que usted accede a ella?

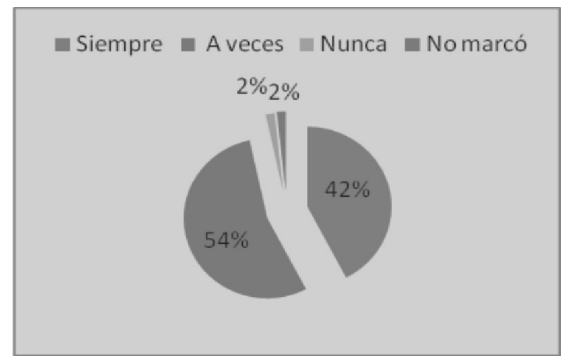

\begin{tabular}{|lcc|}
\hline \multicolumn{1}{|c}{ Variable } & hi & HI \\
\hline Siempre & 50 & $42 \%$ \\
\hline A veces & 64 & $54 \%$ \\
\hline Nunca & 2 & $2 \%$ \\
\hline No marcó & 2 & $2 \%$ \\
\hline TOTAL & 118 & $100 \%$ \\
\hline
\end{tabular}

E1 54\% de los estudiantes encuestados indica que, a veces, la plataforma virtual está habilitada para acceder a ella, el $42 \%$, manifiesta que siempre está habilitada, el $2 \%$ indica que nunca y el otro $2 \%$ se abstuvo de marcar, situación que se convierte en una dificultad, porque la Universidad integra en sus mediaciones pedagógicas el sistema virtual.

Figura 17. ¿En su lugar de residencia tiene fácil acceso a internet con buena velocidad?

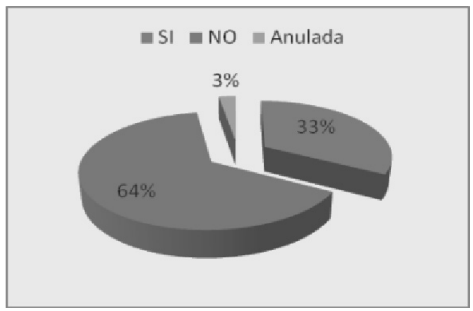

\begin{tabular}{|lcc|}
\hline Variable & hi & HI \\
\hline SI & 39 & $33 \%$ \\
\hline NO & 76 & $64 \%$ \\
\hline Anulada & 3 & $3 \%$ \\
\hline TOTAL & 118 & $100 \%$ \\
\hline
\end{tabular}


De los 118 estudiantes encuestados, el 64\% manifiesta no tener fácil acceso a internet con buena velocidad, un 33\% indica sí tener fácil acceso y un 3\% fue anulado por marcar más de una opción. Se evidencia que la mayoría de los estudiantes no tienen fácil acceso a internet con buena velocidad, hecho que dificulta la adaptación a la metodología a distancia.

Figura 18. ¿De las siguientes herramientas y ambientes virtuales utilizados en el proceso de auto-aprendizaje cuáles se le dificultan más? Señale de 1 a 3 en orden de importancia

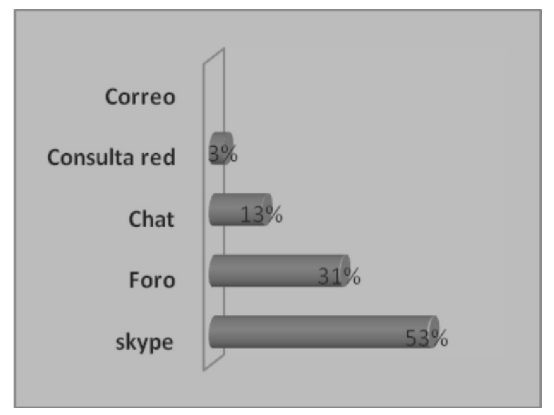

\begin{tabular}{|lll|}
\hline \multicolumn{1}{|c}{ Variable } & \multicolumn{1}{c}{ hi } & \multicolumn{1}{c|}{ HI } \\
\hline skype & 62 & $53 \%$ \\
\hline Foro & 37 & $31 \%$ \\
\hline Chat & 15 & $13 \%$ \\
\hline Consulta red & 4 & $3 \%$ \\
\hline Correo & - & - \\
\hline TOTAL & 118 & $100 \%$ \\
\hline
\end{tabular}

De todos los estudiantes encuestados (118), el 53\% manifiesta, en un primer lugar, tener mayor dificultad con el manejo del ambiente virtual llamado Skype, en segundo lugar, el foro con $31 \%$, en tercer lugar, el chat con $13 \%$ y en un último lugar la consulta en red con un 3\%. En este caso se muestra que a pesar de ser ambientes virtuales que le permite al estudiante utilizar herramientas y desarrollar habilidades de interactuar con la tecnología, aún le hace falta una mayor apropiación y manejo del mismo, se evidencia que los estudiantes tienen dificultades en el manejo de herramientas y ambientes virtuales.

Figura 19. Las estrategias que se usan en la metodología a distancia para que el aprendizaje sea eficaz y significativo

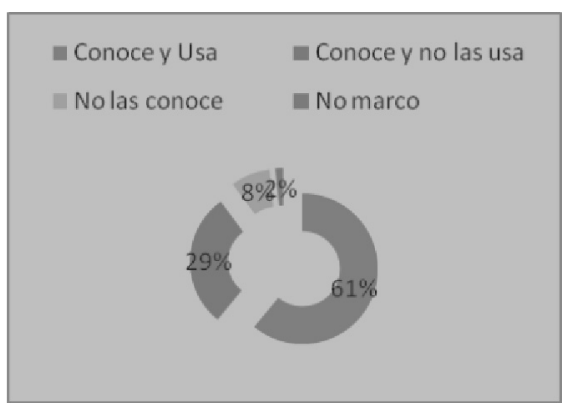

\begin{tabular}{|lcc|}
\hline \multicolumn{1}{|c}{ Variable } & hi & HI \\
\hline Conoce y Usa & 72 & $61 \%$ \\
\hline Conoce y no las usa & 34 & $29 \%$ \\
\hline No las conoce & 10 & $8 \%$ \\
\hline No marcó & 2 & $2 \%$ \\
\hline TOTAL & 118 & $100 \%$ \\
\hline
\end{tabular}

Los estudiantes encuestados de la UNAD, indican en un $61 \%$ que conocen y usan las estrategias de la metodología a distancia, un 29\% manifiesta conocerlas pero no usarlas, el $8 \%$ que no las conoce y un $2 \%$ restante se abstuvo de marcar alguna opción. Se evidencia que los estudiantes no tienen un conocimiento pleno de las estrategias de la metodología a distancia dificultando la adaptación a la misma. 
Figura 20. ¿Qué técnicas de estudio generalmente utiliza en su proceso de aprendizaje?

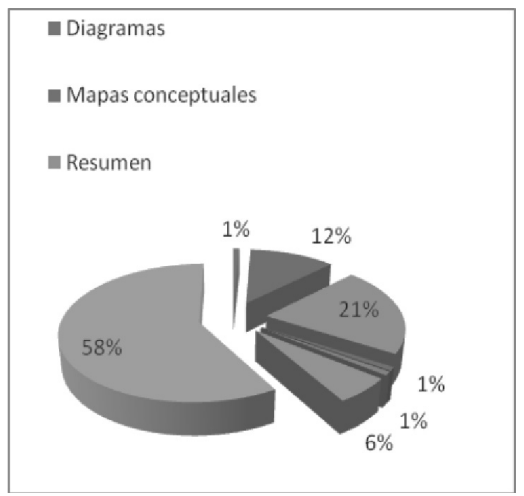

\begin{tabular}{|lll|}
\hline \multicolumn{1}{c}{ Variable } & hi & HI \\
\hline Diagramas & 1 & $1 \%$ \\
\hline Mapas conceptuales & 14 & $12 \%$ \\
\hline Resumen & 25 & $22 \%$ \\
\hline Cuadro sinóptico & 1 & $1 \%$ \\
\hline Ensayos & 1 & $1 \%$ \\
\hline Portafolio & 7 & $7 \%$ \\
\hline $\begin{array}{l}\text { Más de dos (Mapas conceptuales, } \\
\text { resumen, ensayo, portafolio). }\end{array}$ & 69 & $56 \%$ \\
\hline TOTAL & 118 & $100 \%$ \\
\hline
\end{tabular}

Los estudiantes de la UNAD generalmente utilizan más de dos técnicas de estudio (mapas conceptuales, resumen, ensayo y portafolio) en un $56 \%$, un $22 \%$ utiliza resumen, un $12 \%$ los mapas conceptuales, $7 \%$ portafolio, $1 \%$ diagramas, $1 \%$ cuadro sinóptico y un $1 \%$ ensayos.

Figura 21. ¿El cambio del sistema de tutorías presenciales a un sistema virtual representa una dificultad para su aprendizaje?

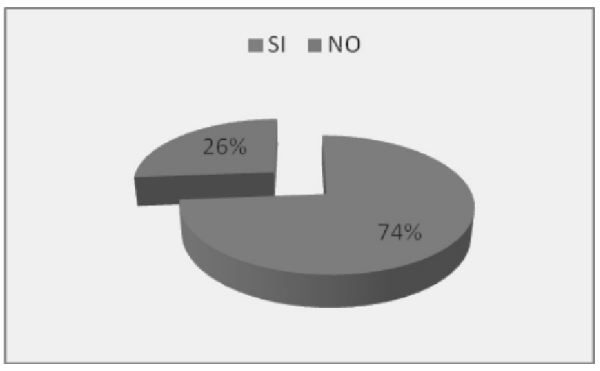

\begin{tabular}{|lcc|}
\hline Variable & hi & HI \\
\hline SÍ & 87 & $74 \%$ \\
\hline NO & 31 & $26 \%$ \\
\hline TOTAL & 118 & $100 \%$ \\
\hline
\end{tabular}

Los estudiantes que fueron encuestados manifiestan en un 74\% que sí presentan dificultad en el cambio de tutorías de sistema presencial a virtual, mientras que un $26 \%$ indica que no tiene dificultad.

Figura 22. ¿Las directivas de la UNAD dan respuesta y o soluciones pertinentes a las dificultades y necesidades académicas del estudiante?

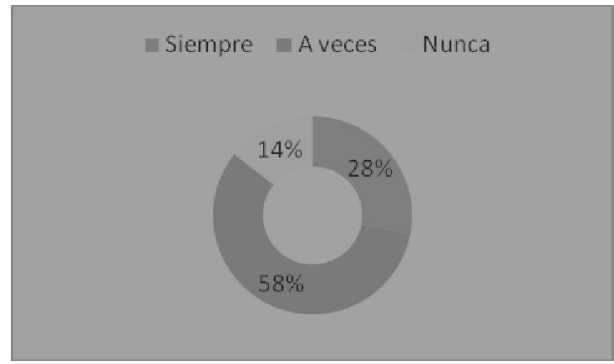

\begin{tabular}{|lcc|}
\hline Variable & hi & HI \\
\hline Siempre & 33 & $28 \%$ \\
\hline A veces & 68 & $58 \%$ \\
\hline Nunca & 17 & $14 \%$ \\
\hline TOTAL & 118 & $100 \%$ \\
\hline
\end{tabular}


Para el 58\% de los estudiantes que fueron encuestados, la UNAD, a veces da respuesta y o soluciones pertinentes a las dificultades, un 28\% indica que siempre dan respuesta y un $14 \%$ que nunca dan respuesta y/o soluciones a los mismos. Situación que evidencia una dificultad, debido a que el estudiante no se siente acompañado.

Figura 23. ¿La UNAD facilita y gestiona el desarrollo de las prácticas dentro de sus cursos académicos?

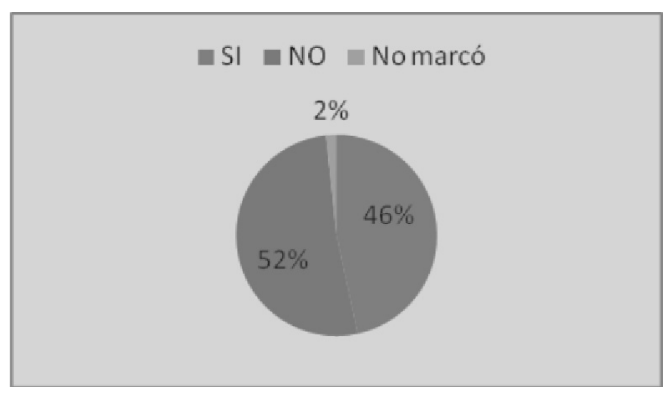

\begin{tabular}{|lcc|}
\hline Variable & hi & HI \\
SÍ & 55 & $47 \%$ \\
NO & 61 & $52 \%$ \\
No marcó & 2 & $2 \%$ \\
TOTAL & 118 & $100 \%$ \\
\hline
\end{tabular}

Más de la mitad (52\%) de los estudiantes que hicieron parte de la encuesta, indica que la UNAD, no facilita, ni gestiona las prácticas académicas. Sin embargo, el (47\%) indica que sí se desarrolla la gestión de las prácticas y el (2\%) se abstuvo de marcar. Se evidencia que los estudiantes se sienten insatisfechos debido a que no se dan las prácticas, situación que desmotiva al estudiante y termina convirtiéndose en una dificultad para la adaptación en la metodología a distancia.

Figura 24. ¿Tiene usted cruce de horarios en sus cursos académicos?

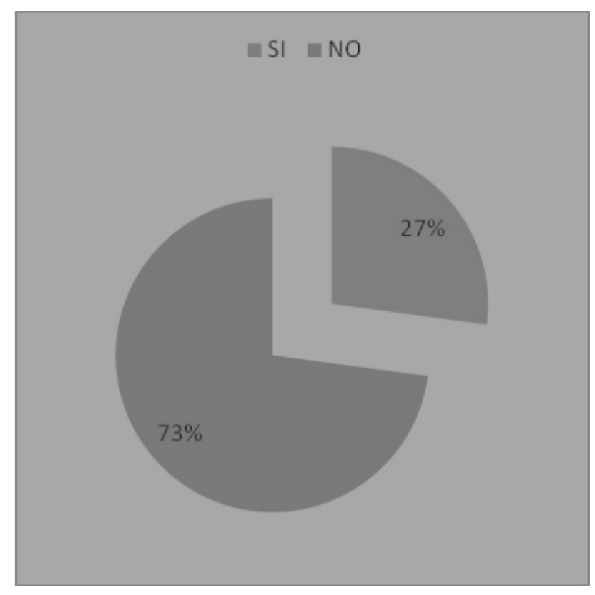

\begin{tabular}{|c|c|c|}
\hline Variable & hi & HI \\
\hline SÍ & 32 & $27 \%$ \\
\hline $\mathrm{NO}$ & 86 & $73 \%$ \\
\hline TOTAL & 118 & $100 \%$ \\
\hline \multicolumn{3}{|c|}{$\begin{array}{l}\text { Cuál: } \\
\text { - Análisis de sistemas, } \\
\text { - Cultura Política, } \\
\text { - Técnicas de Investigación, } \\
\text { - Costos, } \\
\text { - Ecuaciones diferenciales, } \\
\text { - Inglés, } \\
\text { - Ingeniería, } \\
\text { - Telecomunicaciones, } \\
\text { - Herramientas tecnológicas, } \\
\text { - Diseño experimental, } \\
\text { - Seminario, } \\
\text { - Aprendizaje y semillero. }\end{array}$} \\
\hline
\end{tabular}


El 73\% de los estudiantes de la UNAD Ceres La Plata, expresa no presentar dificultades con respecto a cruce de horarios en sus cursos académicos, mientras que el $27 \%$ afirma tener cruce de horarios en algunos cursos académicos.

Los cursos en los cuáles los estudiantes de la UnAD Ceres La Plata manifiestan tener cruce de horarios: Análisis de sistemas, Cultura Política, Técnicas de investigación, Costos, Ecuaciones Diferenciales, Inglés, Ingeniería, Telecomunicaciones, Herramientas Tecnológicas, Diseño Experimental, Seminario, Aprendizaje y con el horario para Semilleros.

Figura 24. Las tutorías orientadas por los profesionales contratados por la UNAD en general

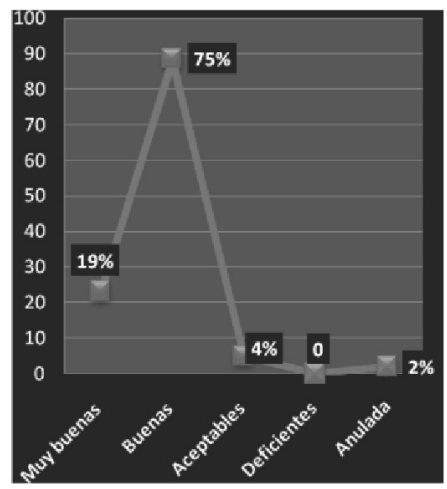

\begin{tabular}{|l|c|c|}
\hline \multicolumn{1}{|c|}{ Variable } & hi & HI \\
\hline Muy buenas & 23 & $19 \%$ \\
\hline Buenas & 88 & $75 \%$ \\
\hline Aceptables & 5 & $4 \%$ \\
\hline Deficientes & - & - \\
\hline Anulada & 2 & $2 \%$ \\
\hline TOTAL & 118 & $100 \%$ \\
\hline
\end{tabular}

Para el 75\% de los estudiantes de la UNAD Ceres La Plata, las tutorías orientadas por los profesionales contratados por la UNAD en general son buenas, para el 19\% son muy buenas, para el $4 \%$ son aceptables y el $2 \%$ fue anulada.

Figura 26. ¿Con qué frecuencia asiste a las tutorías que ve por el sistema tradicional?

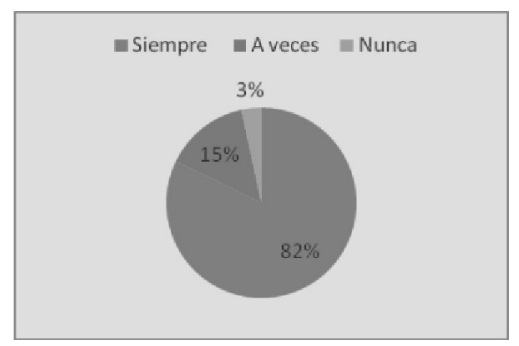

\begin{tabular}{|l|c|c|}
\hline Variable & hi & HI \\
\hline Siempre & 97 & $82 \%$ \\
\hline A veces & 17 & $14 \%$ \\
\hline Nunca & 4 & $3 \%$ \\
\hline TOTAL & 118 & $100 \%$ \\
\hline
\end{tabular}

E1 82\% de los estudiantes de la UNAD Ceres La Plata, manifiesta asistir siempre a las tutorías programadas por el sistema tradicional, el 14\% indica asistir a veces y el 3\% manifiesta que nunca asiste a ellas. De acuerdo con este comportamiento, la mayoría de los estudiantes asisten a tutorías. 
Figura 27. ¿Su tutor cumple con las tutorías programadas?

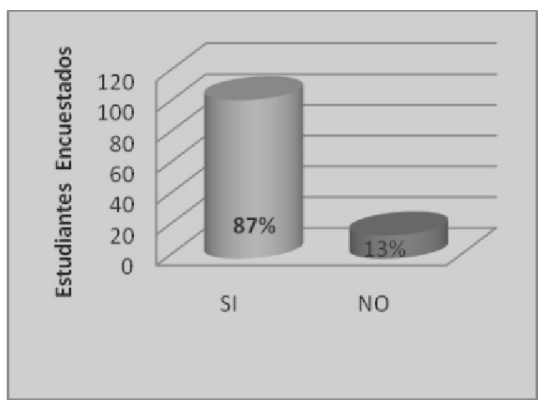

\begin{tabular}{|l|c|c|}
\hline Variable & hi & HI \\
\hline Sí & 103 & $87 \%$ \\
\hline NO & 15 & $13 \%$ \\
\hline TOTAL & 118 & $0 \%$ \\
\hline
\end{tabular}

De los 118 estudiantes de la UNAD Ceres La Plata, el 87\% expresa que sus tutores sí cumplen con todas las tutorías programadas para el período académico y el 13\% manifiesta que los tutores no cumplen con la asesoría. La mayoría de tutores según los estudiantes cumplen con las asesorías, sin embargo, algunos tutores no responden cumplidamente con las asesorías programadas, situación que dificulta y retrasa la adaptación a la metodología a distancia.

Figura 28 ¿En qué curso académico no se cumplió con las tutorías programadas?

Los cursos en los que no se cumplió a cabalidad con la tutoría según los estudiantes fueron: Informática e Hidrología

Figura 29. ¿Presenta retrasos frecuentemente en el inicio de sus tutorías, por la no contratación a tiempo de tutores?

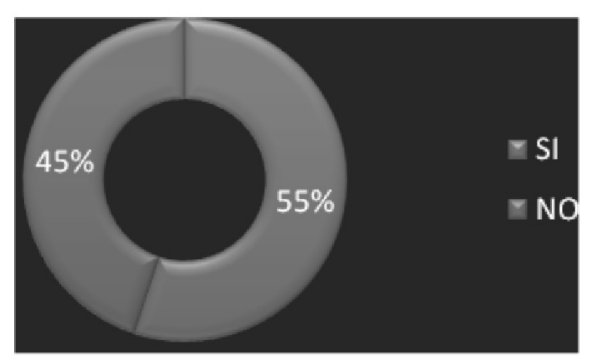

\begin{tabular}{|l|c|c|}
\hline Variable & hi & HI \\
\hline SI & 65 & $55 \%$ \\
\hline NO & 53 & $45 \%$ \\
\hline TOTAL & 118 & $100 \%$ \\
\hline
\end{tabular}

E1 55\% de los estudiantes de la UNAD Ceres La Plata, manifiesta tener retrasos frecuentemente en el inicio de sus tutorías por la no contratación a tiempo de tutores y el $45 \%$ expresa no ser afectado por este hecho. Esta situación desorienta a los estudiantes y les genera insatisfacción en la prestación oportuna del servicio educativo por parte de la universidad, lo cual favorece la presencia de dificultades en la modalidad a distancia. 
Figura 30. La guía de actividades programadas en sus cursos académicos se cumple

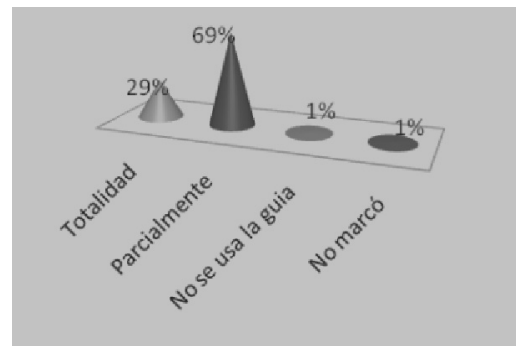

\begin{tabular}{|l|c|c|}
\hline \multicolumn{1}{|c|}{ Variable } & hi & HI \\
\hline Totalidad & 34 & $29 \%$ \\
\hline Parcialmente & 82 & $69 \%$ \\
\hline No se usa la guía & 1 & $1 \%$ \\
\hline No marcó & 1 & $1 \%$ \\
\hline TOTAL & 118 & $100 \%$ \\
\hline
\end{tabular}

Según lo manifestado por los estudiantes de la UNAD Ceres La Plata, la guía de actividades programadas en sus cursos académicos se cumple parcialmente $(69 \%)$, se cumple en su totalidad (29\%), no se usa la guía (1\%), no marcó (1\%). Este aspecto genera en los estudiantes y en los tutores una dificultad de programación y orientación, ya que no se hace una implementación y seguimiento de la guía, no hay una estrategia de interacción y comunicación efectiva, entre los actores durante todo el proceso de aprendizaje. Y el estudiante no tiene una apropiación efectiva y pertinente de los conocimientos determinado por cada curso académico, y por lo tanto, no se realiza una verdadera asesoría y en consecuencia una efectiva formación profesional.

Figura 31. El tiempo diario que le dedica a su aprendizaje por curso académico

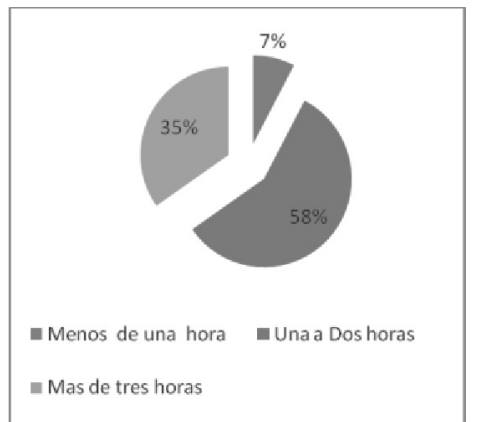

\begin{tabular}{|l|c|c|}
\hline Variable & hi & HI \\
\hline Menos de una hora & 9 & $8 \%$ \\
\hline Una a dos horas & 68 & $58 \%$ \\
\hline Más de tres horas & 41 & $35 \%$ \\
\hline TOTAL & 118 & $100 \%$ \\
\hline
\end{tabular}

El tiempo diario que los estudiantes de la UNAD Ceres La Plata, le dedican a su aprendizaje, es un 58\% de una a dos horas; un 35\% más de tres horas y un $8 \%$ menos de una hora . Este comportamiento muestra que los estudiantes no están aplicando el tiempo requerido para obtener un buen desarrollo del aprendizaje auto-dirigido creando así una dificultad para la apropiación del conocimiento. 
Figura 32. ¿Desarrolla y entrega las actividades a tiempo a su tutor?

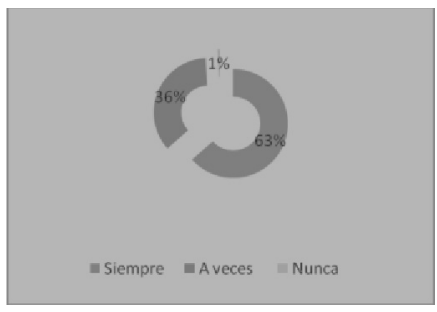

\begin{tabular}{|l|c|c|}
\hline Variable & hi & HI \\
\hline Siempre & 75 & $64 \%$ \\
\hline A veces & 42 & $36 \%$ \\
\hline Nunca & 1 & $1 \%$ \\
\hline TOTAL & 118 & $100 \%$ \\
\hline
\end{tabular}

El 64\% de los estudiantes de la UNAD Ceres La Plata, manifiesta que siempre desarrolla y entrega las actividades a tiempo a su tutor, el $36 \%$ a veces las entrega a tiempo y el $1 \%$ nunca las desarrolla ni las entrega a tiempo.

Figura 33. ¿Se le dificulta el desarrollo de las actividades programadas en los cursos académicos que ve por plataforma virtual?

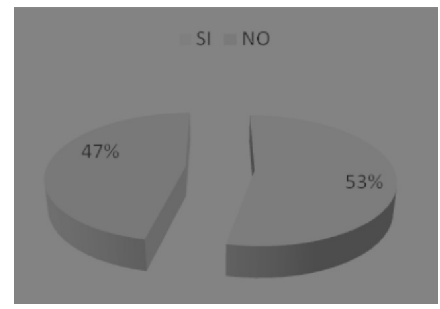

\begin{tabular}{|l|c|c|}
\hline Variable & hi & HI \\
\hline SI & 63 & $53 \%$ \\
\hline NO & 55 & $47 \%$ \\
\hline TOTAL & 118 & $100 \%$ \\
\hline
\end{tabular}

Al $53 \%$ de los estudiantes de la UNAD Ceres La Plata, se le dificulta el desarrollo de actividades programadas en sus cursos académicos que ve por plataforma virtual y al $47 \%$ no se le dificulta realizar dichas actividades por plataforma virtual, indicando con esto que a pesar de estar matriculados en un sistema virtual, no se han familiarizado con la misma, lo que genera una dificultad para el cumplimiento de sus deberes académicos.

Figura 34. ¿Conoce el plan de estudio de su programa?

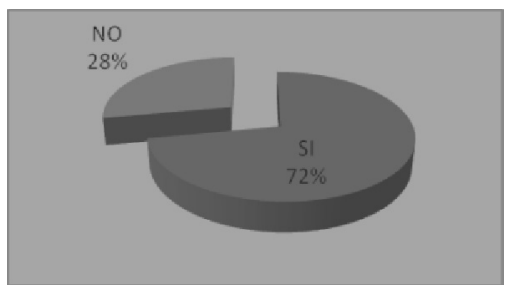

\begin{tabular}{|l|c|c|}
\hline Variable & hi & HI \\
\hline SÍ & 85 & $72 \%$ \\
\hline NO & 33 & $28 \%$ \\
\hline TOTAL & 118 & $100 \%$ \\
\hline
\end{tabular}

Del $100 \%$ de los estudiantes de la UnAD Ceres La Plata, el $72 \%$ conoce el plan de estudio de su programa de pregrado y el $28 \%$ manifiesta no conocerlo. Este $28 \%$ representa un alto 
porcentaje, pues los estudiantes deben conocer su plan de estudIos para matricular sus cursos académicos.

Figura 35. ¿La programación de su plan de estudio se ajusta al periodo académico que cursa?

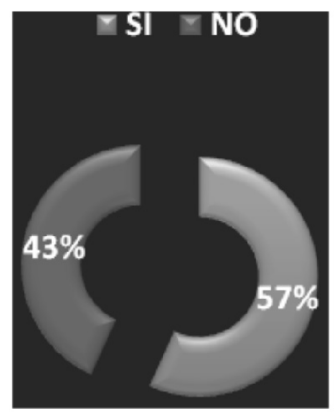

\begin{tabular}{|l|c|c|}
\hline Variable & hi & HI \\
\hline SÍ & 67 & $57 \%$ \\
\hline NO & 51 & $43 \%$ \\
\hline TOTAL & 118 & $100 \%$ \\
\hline
\end{tabular}

El 57\% de los estudiantes de la UNAD Ceres La Plata, manifiesta que la programación de su plan de estudio se ajusta al período que cursa y el $43 \%$ expresa que su plan de estudio no se ajusta a su periodo académico, evidenciando que los estudiantes no tienen un conocimiento pleno de plan de estudio general por programa.

\section{¿Qué otra dificultad constante encuentra en el desarrollo de sus cursos académicos?}

- Cruce de horarios

- Difícil acceso a internet banda ancha

- No devuelven las tutorías

- Tutorías virtuales no comprometidas con el curso académico

- Escaso manejo de la plataforma virtual

- Falta de prácticas en los cursos teórico-prácticos

- Acceso a implementos tecnológicos

- Módulos desactualizados

- Falta de retroalimentación en las evaluaciones

- Trabajos de grupo colaborativo por plataforma virtual

- Eventos en horas de tutorías

- Poca disponibilidad por parte de algunos tutores

- Carencia de internet con buena velocidad en municipios aledaños en los cuales la UNAD tiene estudiantes (Páez, Inzá, Itaibe, Paicol, La Argentina, Rio Negro, Nátaga, Gigante), e incluso en algunos sitios no hay acceso a este medio.

- La no existencia de fuentes bibliográficas

- La oferta de cursos electivos sin ninguna profundización para el estudio que se está desarrollando

- Como sugerencia ampliar el horario de la sala de internet 


\section{CONCLUSIONES}

En los estudiantes de los diversos programas de pregrado, que oferta la Universidad Nacional Abierta y a Distancia unAD, Ceres Villa de San Sebastián de La Plata, Huila, se identificaron dificultades de adaptación al sistema de educación a distancia, ya sea por el sistema virtual o tradicional, que imparte esta institución de educación superior, afectando el proceso de auto aprendizaje y continuidad de los estudiantes en su formación tecnológica y profesional.

Las estrategias instituidas en el Ceres Villa de San Sebastián de La Plata y dirigidas a la comunidad Unadista, para que asimile y se adapte a la metodología a distancia, presenta falencias en su ejecución, situación reflejada en los bajos resultados académicos, desmotivación, suspensión parcial o definitiva de la actividades académicas en los estudiantes; en los tutores la incompleta aplicación de la metodología a distancia, evidenciada en las observaciones a las tutorías.

En el desarrollo de este proyecto de investigación se encontraron limitaciones de tiempo y espacio para la recolección de información, que retardaron el proceso de análisis de datos.

Los programas de pregrado, de las escuelas: Ciencias Contables, Administrativas, Contables y de Negocios ECAEN, Escuela de Ciencias Sociales Artes y Humanidades ECSAH, Escuela de Ciencias Básicas Tecnología e Ingeniería ECBTI, Escuela de Ciencias Agrarias, Pecuarias y de Medio Ambiente ECAPMA, que oferta el Ceres La Plata, presentan en general las mismas dificultades, que afectan al proceso de auto aprendizaje, asimilación y adaptación a la metodología impartida por la universidad.

A criterio de los investigadores el ser egresado del sistema de educación presencial, aumenta el grado de dificultad en la adaptación a la metodología a distancia de los estudiantes, y más cuando no encuentra claridad y no se induce e instruye correctamente en su rol como sujeto activo de su propio aprendizaje.

Los estudiantes, en general, expresan su preferencia por el sistema tradicional, pues consideran que aún no se encuentran lo suficientemente instruidos y familiarizados con la mediación pedagógica virtual, en su gran mayoría acceden a esta con alto grado de dificultad. Lo anterior sumado a los problemas de conectividad y el acceso fácil a internet con buena velocidad en sus lugares de residencia.

Un porcentaje del 53\% de los estudiantes encuestados expresó estar satisfecho con la formación académica recibida por parte del Ceres Villa de San Sebastián de La Plata y la población restante el $47 \%$ se mostró insatisfecha lo que implica revisión de estrategias que busquen la satisfacción de las expectativas académicas de los estudiantes.

Una dificultad evidente son los costos de la matrícula y otros dentro de los cuales se pueden enunciar: transporte a la sede para los estudiantes que residen en zonas rurales y otros municipios, gastos de impresión de trabajos escritos, de actividades a desarrollar y de 
contenidos de los módulos, cuando no se cuenta con PC personal, alquiler del servicio de internet, pues los equipos de la Universidad son insuficientes para atender a los estudiantes de manera permanente, especialmente cuando se concentran todos los estudiantes en días de tutorías.

El estudiante como responsable de sus procesos académicos y sujeto activo de su aprendizaje, no demuestra compromiso con sus deberes académicos. Es así como el 100\% no asiste, como debería ser, al evento de inducción y re inducción programados al inicio de actividades de cada período académico; también la inasistencia frecuente, aunque en un porcentaje no superior al $50 \%$, a las tutorías por sistema tradicional; desconocimiento de sus planes de estudios.

El porcentaje de los estudiantes que no maneja los programas básicos de informática, es significativamente alto, $25 \%$, pues estos programas se consideran fundamentales para el desarrollo de las actividades académicas diarias; es así como, representa una dificultad importante que no le permite adaptarse fácilmente a la metodología a distancia.

Los problemas de conectividad representan un grado alto de dificultad en los estudiantes, pues no hay fácil y eficiente acceso a internet, según un $64 \%$ de los encuestados y la plataforma virtual de la Universidad algunas veces se encuentra inhabilitada. De igual manera, existe la dificultad en el manejo de herramientas y ambientes virtuales, en primer lugar del Skype, en segundo lugar, el foro y en tercer lugar el chat.

La escasa gestión de la UNAD como facilitadora y puente, para el desarrollo de prácticas en los cursos académicos en los diversos contextos, según el programa de pregrado, genera insatisfacción en el estudiante y representa para él una dificultad en la formación integral como futuro profesional. Los estudiantes solicitan a su vez respuesta y soluciones pertinentes a sus necesidades académicas.

Otra dificultad frecuente es el retraso en el inicio de tutorías por la no contratación a tiempo de tutores, ya que el estudiante en muchos casos no se apropia de sus procesos académicos, no asiste siempre al evento de inducción, por lo tanto, inicia desorientado y con la incertidumbre de cuándo empezar sus estudios.

Actualmente, en la UNAD y a raíz de los resultados preliminares de la presente investigación, se han venido implementando estrategias para mejorar aspectos como: inducción - re inducción, y acompañamiento a los estudiantes por el encargado de la sala de computo.

Para los estudiantes, el desarrollo parcial de la guía de actividades genera insatisfacción, pues considera que el proceso académico no se cumple a cabalidad. Esto debido a que las tutorías se ven afectadas con eventos no programados. de lo que se puede concluir como una situación que desmotiva a los estudiantes convirtiéndose en una dificultad. 


\section{REFERENCIAS BIBLIOGRÁFICAS}

ALARCÓN, José Guillermo, Competencias pedagógicas autoevaluación docente.

FANDIÑO, Graciela. Nuevas tendencias de la educación en Colombia.

LEAL AFANADOR, Jaime Alberto, y otros, Inducción Unadista Educación para Todos.

MONREAL, José Luis, Editorial Océano, Diccionario de Sinónimos y Antónimos.

MALDONADO GRANADOS, Luis Facundo, Gestión del conocimiento visibilidad del desarrollo científico.

MALDONADO GRANADOS, Luis Facundo. Gestión del conocimiento Visibilidad del Desarrollo Científico. Revista UNAD 25 años núm. 001.

SANABRIA, BETTER. (1994), Ministro de Educación Nacional, Memorias un año de cambio en la educación colombiana. En: Revista Cultural N.0 7, Inventario.

\section{DOCUMENTOS}

PAPS Proyecto Académico Pedagógico Solidario

Documento Contratación de Tutores

Información de Matriculas por periodo académico 2007-2008-2009

\section{WEB BIBLIOGRAFÍA}

www.byd.com.ar/ed11

WWW. edutec.rediris.es/

www. bvs.sld.cu/revistas/aci/vol11_1_03/aci02103.htm

www.virtual.org

www.wikipedia.com

www.slideshare.net

www.uoc.edu/rusc

www.aulaglobal.net.ve

www.mineducacion.gov.co

www.occidente30dias.gov.co

www.unad.edu.co lindex.php

www.gobernacionhuila.gov.co

www.mineducación .gov.co/1621/articles-85906_archivo_pdf

www.mineducacion.gov.co/1621/articles-85860_archivo_pdf.pdf

www.mineducación.gov.co/1621/articles-85860_archivo_pdf 